\title{
An adaptive weight value-based multi-scale retinex algorithm for color image enhancement
}

\author{
QianshunLiu ${ }^{\mathrm{a}}$, Jian Bai ${ }^{\mathrm{b}}$ and Feihong $\mathrm{Yu}^{\mathrm{c}}$
}

\author{
College of optical science and engineering, Zhejiang University, Hangzhou310058, China \\ aliuqianshun_zju@163.com, bbai@zju.edu.cn, cfehong@zju.edu.cn
}

Keywords: Retinex,HDR compression, color image enhancement.

\begin{abstract}
There are limited dynamic range problems when real world scenes are captured from a digital camera and displayed on monitors.Retinex is one of the most popular theories for dynamic range compression, color constancy and color rendition.However, there still exist some problems, such as hue shift and excessive noise, especially for those images including large bright areas and dark regions.In order to solve these problems,an adaptive weight value-based multi-scale retinex algorithm is proposed in this paper. Compared to other methods, visual experiments and data evaluation show that the proposed algorithms can achieve better performance for color image enhancement and noise suppression simultaneously. What is more, there is no need for color correction phase in the proposed algorithm, which makes it more efficient and convenient to apply.
\end{abstract}

\section{Introduction}

The real world luminance range is much larger than those of digital cameras and display devices. While, the dynamic range of digital camerasis much more limited.Thus, images captured by a digital camera are shown differently from the human perception for the same scenes. To solve this problem, many tone mapping algorithms have been developed for rendering the high dynamic range (HDR) images on low dynamic range (LDR) devices.Among them, retinex theory is one of the most frequently used methods, which was developed by Land [1] as a model of lightness and color perception of human vision. According to the principle of retinex theory, an image can be defined as

$$
I(x, y)=L(x, y) \bullet R(x, y)
$$

Where $I(x, y)$ is the amount of light human eyes perceive, $L(x, y)$ represents the illumination, and $R(x, y)$ is the reflectance which contains the object's characteristics.The typical method of retinex theory is the center/surround algorithm which calculates the difference in the log-domain between center pixel and the weighted average value of its surrounds [2]. Jobson et al. proposed the single-scale retinex (SSR) and multi-scale retinex (MSR), which are expressed as

$$
\begin{gathered}
R_{i}^{S S R}(x, y)=\log I_{i}(x, y)-\log \left[G(x, y) * I_{i}(x, y)\right] \\
R^{M S R}(x, y)=(1 / N) \cdot \sum_{i=1}^{N} R_{i}^{S S R}
\end{gathered}
$$

Where $R_{i}^{S S R}(x, y)$ is the retinex output, $I_{i}(x, y)$ is the image pixel of the $i$-th color channel, " $*$ ” denotes the convolution operation, and $G(x, y)$ represents the Gaussian function.The MSR is the average weighted sum of several SSRs withdifferent Gaussian filter size.However, MSR is not desirable for color images because the RGB channel is unbalanced due to the SSR processing for each RGB component. As a result, the MSR processing causes the gray-out of the image in entire or specific regions.Besides, retinex deals with R, G, and B channels separately, accordingly there are always color artifacts that exaggerate hue shift and desaturation [3]. Thus, color space that can distinguish the luminance and chrominance components is needed to preserve the hue and saturation of captured images, such as HSV. In recent years, there are many researchesproposed to improve MSR in terms of natural impression [4-5].Although these algorithms have achieved amazing performance on visual perception, there still exist some drawbacks, such as failing to produce natural images because of some color problems or too much noise appeared due to over-enhancement of the 
dark regions. The most important reason is that most of these algorithms use the average weight (always 1/3) for different scale outputs. Thus, the noise in dark areas is over amplified when enhancing the image contrast.To solve these problems, an adaptive weight value-based multi-scale retinex (AVBMSR)is proposed for color image enhancement and noise suppression in this paper.Visual experiments and data evaluation show that the proposed algorithms can produce natural and appealing enhanced images without color correction phase. More importantly, the noise appeared in dark regions can be suppressed in an acceptable degree.

\section{The proposed algorithm}

By combining the Eq. (2) and Eq. (3), the original form of the MSR can be expended as follows:

$$
R^{M S R}=\frac{1}{3} \log \frac{I_{i}}{G_{1} * I_{i}}+\frac{1}{3} \log \frac{I_{i}}{G_{2} * I_{i}}+\frac{1}{3} \log \frac{I_{i}}{G_{3} * I_{i}}
$$

As explained above, HSV color space is often used in computer vision because it can separate the luminance component and chrominance components. What is more, because only one channel is processed and there is no need of color correction, it can reduce the computation time.Thus, we extracts the value channel in HSV color space to preserve the hue and saturation, sowe can get

$$
R^{\mathrm{VBMSR}}=\frac{1}{3} \log \left(\frac{I_{v} \cdot I_{v} \cdot I_{v}}{\left(\mathrm{G}_{1} * I_{v}\right) \cdot\left(\mathrm{G}_{2} * I_{v}\right) \cdot\left(\mathrm{G}_{3} * I_{v}\right)}\right)
$$

Where $I_{v}$ represents the value channel in HSV color space of the original image. $G_{s}(\mathrm{~s}=$ 1,2,3)represents the Gaussian low-pass filter with scale 15, 80 and 250 respectively, andalmost all related studies use these values.Usually, the three weights are set to be the average value of scales. It means $1 / \mathrm{N}=1 / 3$ in Eq. (3), just as MSR, LBMSR and many other approaches did. However, this average weight will cause desaturation problem in bright areas and amplify noise in dark regions. Thus, we combine different SSR outputs in a way that theweight associated with each SSR scale is adaptivelycomputed from the content of the input image, defined as follows:

$$
\begin{gathered}
p_{s}(x, y)=\exp \left[\left(-I_{v}(\mathrm{x}, \mathrm{y})-\mu_{s}\right)^{2} / 2 \sigma^{2}\right] \\
\omega_{s}(x, y)=p_{s}(x, y) / \sum_{s=0}^{3} p_{s}(x, y)
\end{gathered}
$$

Where $p_{s}(s=1,2,3)$ represents the likelihood probability associated with each SSR scale and $\omega_{s}(s=1,2,3)$ is the corresponding adaptive weight. The four Gaussian distributions represent dark-pixel class, medium-dark-pixel class, medium-bright pixel class, and bright-pixel class, with their Gaussian mean values being equally spaced in the luminance range $[0,255]$ and are empirically set as $\mu_{0}=32, \mu_{1}=96, \mu_{2}=160$ and $\mu_{3}=224$. The standard deviation of each class is defined as the same value $\sigma=32$. In addition, for producing a natural output image, the input image is combined with every SSR output. To this end, we initially set $p_{0}=1$ which is used to compute the weight $\omega_{0}$ associated with the input image. Thus, we change the Eq. (5) to Eq. (8), shown as follows:

$$
R_{v}^{\text {AVBMSR }}=\log \left(\frac{I_{v}^{\omega_{1}+\omega_{2}+\omega_{3}}}{\left(G_{1} * I_{v}\right)^{\omega_{1}} \cdot\left(G_{2} * I_{v}\right)^{\omega_{2}} \cdot\left(G_{3} * I_{v}\right)^{\omega_{3}}}\right)+\omega_{0} I_{v}
$$

Although noise in dark regions can be reduced using Eq. (8), the global brightness of the result will be too low to recognize some details. An effective solution is to add parameters to improve its impression, as shown in Eq. (9).

$$
R_{v}^{\mathrm{ALVMSR}}=\log \left(\frac{\alpha I^{\omega_{1}+\omega_{2}+\omega_{3}}}{\left(G_{1} * I\right)^{\omega_{1}}+\left(G_{2} * I\right)^{\omega_{2}}+\left(G_{3} * I\right)^{\omega_{3}}}\right)+\beta \omega_{0} I
$$


The result of the above processing will have both negative and positive pixel values. Thus, the simplest color balance (SCB) method is adopted to map the pixels into the output range, as expressed in Eq. (10).

$$
R_{v}(x, y)=\left\{\begin{array}{cc}
255, & R_{v}(x, y)>P_{99} \\
255 \times \frac{R_{v}(x, y)-P_{1}}{P_{99}-P_{1}}, & P_{1} \leq \mathrm{R}_{v}(x, y) \leq P_{99} \\
0, & \mathrm{R}_{v}(x, y)<P_{1}
\end{array}\right.
$$

Where $P_{1}$ and $P_{99}$ denote the 1-th and 99-th percentile, which is defined as the value where 1 and 99 percent of the output values, and $R_{v}(x, y)$ represents $R_{v}^{A V B M S R}$ in Eq.(9).

To obtain the final enhanced image, we only need to replace the original value channel using the output of Eq. (10). Unlike most of methods, there is no need of color correction phase which is at risk of inverting colors in the image.

\section{Experimental results and data evaluation}

In this section, we first demonstrate the outputs of different Gaussian scales and its adaptive weight maps. Then, we compare the proposed algorithms with other six methods from a visual standpoint. Finally, four metrics are employed to evaluate the experimental results in an objective way.

In the first experiment, Fig.1 gives an example showing the output images of different scales.In order to show a color image, each output of is merged with the $\mathrm{H}$ and $\mathrm{S}$ channels of the original input image.

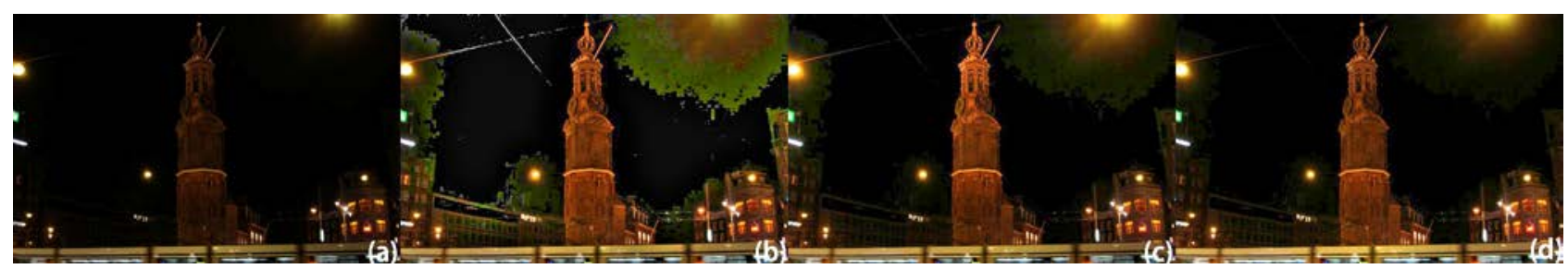

Fig. 1 The outputs of different scales (a) original (b) $\sigma=15$ (c) $\sigma=80$ (d) $\sigma=250$.

From Fig.1(b), we can see that the local contrast can be enhanced by using small-scale whereas some artifacts appear along the tower. More important, it produces too much noise in dark areas. On the contrary, these problems does not appear in the output of large-scale, but enhancement of some details are not enough. Based on this fact, we should give a large weight value to small-scale for those image detail pixels and give a large weight value to large-scale for those dark or bright pixels. Fig.2shows the different weight maps, which indicate the weight values for each image pixel, associated with each scale for the image shown in Fig.1.

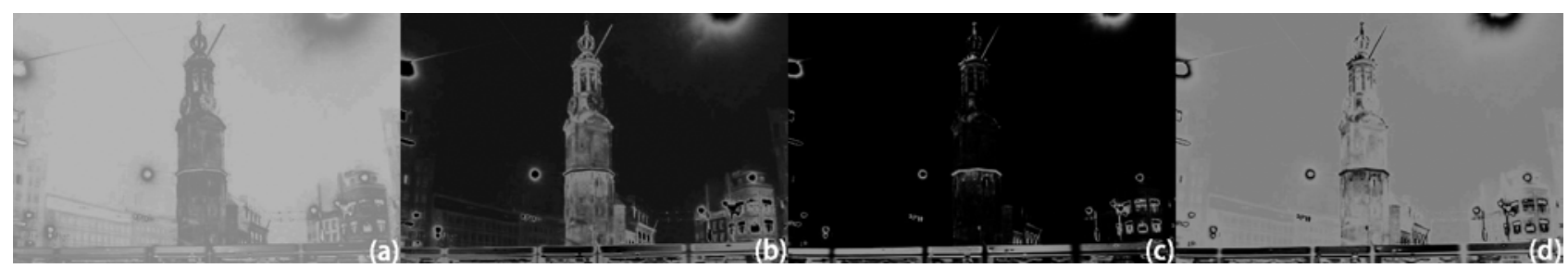

Fig. 2Weight maps associated with original V channel of the input image and otherscale outputs.

As seen in Fig.2, different pixels have different weight values, which is dependent on pixel value of the input image. Further, we can see that the weight map associated with small-scale exhibits large weights for those pixels in fine detail regions (please see Fig.2(b)), whereas the weight map associated with large-scale or $\mathrm{V}$ channel of the input image presents large weights for those pixels in large dark areas (please see Fig.2(d) and Fig.2(a)). In the following experiment, we compare the enhanced results of Fig.1(a) by our proposed algorithms with other two methods, as shown in Fig.3. 


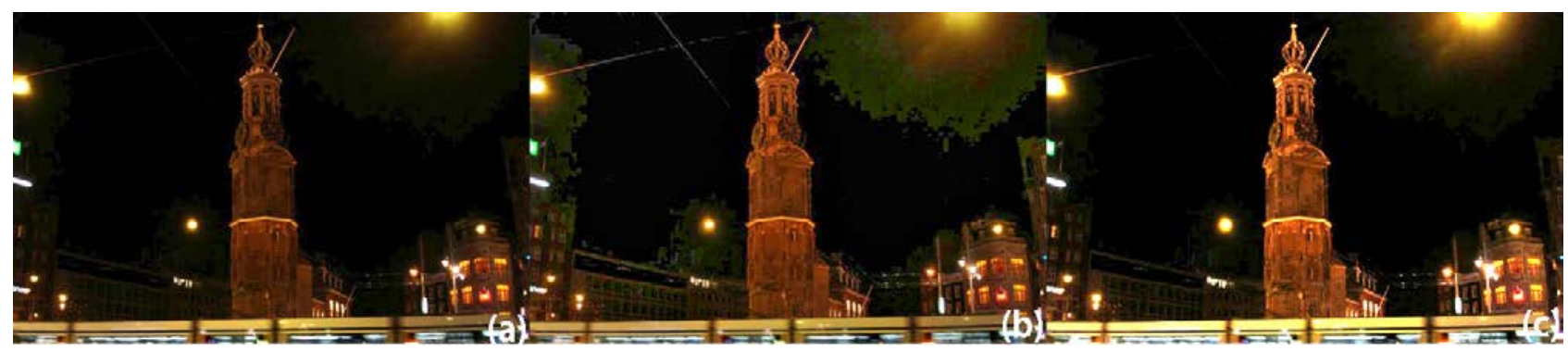

Fig. 3 Comparison of the enhanced results (a) LBMSR (b) AMSR (c) AVBMSR ( $\alpha=10, \beta=0.8$ )

As seen in Fig.3, the proposed AVBMSR algorithm obtains a better enhancement effect, although both of them are based on retinex theory.In order to assess the visual quality of the results in an objective way, four metrics are employed here. They are Peak Signal to Noise Ratio (PSNR), Structural Similarity (SSIM) [6], and Visual Contrast Measure (VCM) [7].

Table 1 Results of the PSNR, SSIM and VCM metrics for Fig.3

\begin{tabular}{ccccc}
\hline Fig.3 & PSNR & SSIM & VCM & EC \\
\hline (a) & 18.08 & 0.7270 & 61.11 & 0.1524 \\
(b) & 20.08 & 0.6519 & 78.88 & 0.1603 \\
(c) & $\mathbf{2 2 . 1 5}$ & $\mathbf{0 . 8 3 9 4}$ & $\mathbf{8 8 . 0 0}$ & $\mathbf{0 . 1 3 1 2}$ \\
\hline
\end{tabular}

In Table 1, the best results of each metric are shown in bold. From the data evaluation,the proposed algorithmcan achieve the best visual effect and image quality.

\section{Summary}

Present tone mapping algorithms may cause several problems, especially for those images containing large bright and dark regions. To solve this problem, the algorithm AVBMSR with adaptive weight is proposed in this paper. Visual experiments and data evaluation show thatthe proposed algorithm can produce natural and appealingenhanced images without color correction. More importantly, the noise appeared in dark regions can be suppressed in an acceptable degree. As a result, the proposed algorithmcan achieve better performance in color enhancement and noise suppression simultaneously.

\section{References}

[1]. Land, E. H. An alternative technique for the computation of the designator in the retinex theory of color vision. Proceedings of the National Academy of Sciences. Vol. 83 (1986) No. 10, p. 3078-3080.

[2]. Jobson, D.J., Rahman,Z.U.,Woodell, G. Properties and performance of a center/surround retinex. Image Processing, IEEE Transactions on. Vol. 6 (1997) No.3, p.451-462.

[3]. Kimmel R, Elad M, Shaked D, et al. A variational framework for retinex. International Journal of computer vision.Vol. 52 (2003) No.1, p.7-23.

[4]. Lee C H, Shih J L, Lien C C, et al. Adaptive Multiscale Retinex for Image Contrast Enhancement. Signal-Image Technology \& Internet-Based Systems.Japan, 2013, p.43-50.

[5]. Sun B, Tao W, Chen W, et al. Luminance based MSR for color image enhancement. Image and Signal Processing Congress on.China,2008, p.358-362.

[6]. Wang Z, Bovik A C, Sheikh H R, et al. Image quality assessment: from error visibility to structural similarity. Image Processing, IEEE Transactions on.Vol.13 (2004) No.4, p.600-612.

[7]. Jobson D J, Rahman Z, Woodell G A. Statistics of visual representation. International Society for Optics and Photonics. America, 2002, p.25-35. 